\title{
Detection of partial cDNA sequences differentially expressed in patients with myelodysplasia
}

\begin{abstract}
The most common chromosomal aberrations in myelodysplastic syndromes (MDS) are complete or partial loss of chromosomes 5 and 7, and trisomy 8. To identify genes important in the pathogenesis of this disease that could be associated with these gross chromosomal defects, we have employed the differential display PCR (DDPCR) procedure developed by Liang and Pardee. This method allows simultaneous comparison of several cDNA sources for the presence of differentially expressed genes. Polymorphonuclear cells (PMNs) from two MDS patients, containing a $5 q$ deletion or a trisomy 8 , and three healthy controls were used. Initial screening resulted in the identification of five and three partial cDNA sequences, respectively that were either differentially expressed in both patient samples or in individual patients, as compared with the controls. The authenticity of aberrant expression was verified by reanalyzing the same primer combinations on newly prepared cDNA. Differential expression of the three remaining fragments was subsequently checked on a larger panel of MDS patients, using amplicon-specific primer sets. These were obtained by cloning and sequencing of the fragments. For one partial cDNA (DC3), the original expression pattern, i.e., decreased expression in individual MDS patients, was confirmed. These results demonstrate the utility of the DDPCR procedure to isolate differentially expressed sequences in primary patient samples where the availability of cells is a limiting factor.
\end{abstract}

M.J.P.L. Kroef · R. Willemze · J.E. Landegent (西)

Laboratory of Experimental Hematology, Building 1 C2-R, Leiden University Hospital, P.O. Box 9600,

NL-2300 RC Leiden, The Netherlands

J.H. Jansen

Laboratory of Hematology, Erasmus University, Rotterdam, The Netherlands

This study was supported by a grant from the J.A. Cohen Institute for Radiopathology and Radiation Protection
Key words Differential display - Myelodysplastic syndromes $\cdot$ Chromosomal aberration

\section{Introduction}

Myelodysplastic syndromes (MDS) are a heterogeneous group of clinically defined disorders characterized by ineffective hematopoiesis and involving at least two myeloid lineages. Patients with MDS show peripheral blood cytopenia and a normocellular or hypercellular bone marrow with signs of defective maturation. A classification into five subgroups has been made by the French-American-British (FAB) Cooperative Study Group, based on the number of blast cells in peripheral blood and bone marrow and the number of peripheral blood monocytes [1]. Chromosomal abnormalities are observed in the bone marrow of $40-50 \%$ of MDS patients unrelated to previous therapy [11]. The most common abnormalities are loss of a part of chromosome $5 q$, monosomy 7 , and trisomy 8 .

It is generally accepted that the MDS are clonal disorders involving myeloid cells. However, X-chromosome inactivation studies on the clonal nature of lymphopoiesis have shown ambiguous results. Some reports have described a clonal involvement of lymphoid cells [17], whereas we have demonstrated that T lymphocytes, B lymphocytes, and natural killer cells are polyclonal [18]. Furthermore, studies on the occurrence of chromosome 5, 7, and 8 aberrations have demonstrated that they are also restricted to cells of myeloid origin [5-7].

Because the most common genetic defects in MDS involve gross chromosomal aberrations, it is extremely difficult to identify the gene(s) which might play a role in the pathogenesis of the disease. In case of the $5 q$ deletions, the critical region defined at 5q31-q33 [8] is still too large to allow pinpointing of the responsible tumorsuppressor gene(s). Moreover, a simple genetic approach to analyzing the aneuploidies is not at hand. An alternative procedure would be analysis of differences 
in gene expression between normal and aberrant cells. This can be accomplished by the conventional subtractive hybridization technique [9] that has, for example, been successfully applied to identify maspin, a gene with tumor-suppressing activity in human mammary epithelial cells [20]. However, this method allows comparison of only two cell sources per experiment and require relatively large amounts of RNA. These drawbacks can be overcome by using the differential display PCR (DDPCR) procedure [10], which has allowed the identification of aberrantly expressed genes in breast and brain tumors $[14,15]$. The general strategy of this method is PCR amplification of subsets of cDNA, as obtained by reverse transcription of total RNA with anchored poly $(\mathrm{dT})_{12}$ primers. As PCR primers, the appropriate anchored poly $(\mathrm{dT})_{12}$ and short (10-mers) random oligomers are used. The resulting expression patterns obtained from various cell sources are simultaneously analyzed by displaying the generated short cDNA fragments on denaturing PAA gels.

In order to test its potential application in MDS, we have performed a pilot study to detect differentially expressed genes in two MDS patients, one with a chromosome 5 deletion and the other with a trisomy 8 , as compared with control samples from three healthy individuals. As cell sources, purified polymorphonuclear cells (PMNs) were used, since they were shown to contain the genetic defects $[6,7]$. Both partial cDNA sequences that were differentially expressed in the individual patients or aberrantly expressed in both patients were analyzed. Because of the limited material available, Northern analysis could not be applied to unambiguously show aberrant expression of a given fragment. Several PCR-based control experiments were therefore included to confirm the authenticity of differential expression.

\section{Materials and methods}

Patients

Two MDS patients were analyzed. The first patient (UPN 1) was a 67-year-old women, who suffered from refractory anemia (RA). Cytogenetic analysis on GTG-banded metaphases demonstrated a deletion of the long arm of chromosome 5 in $90 \%$ of her bone marrow cells. The other patient (UPN 2), a 43-year-old man, was diagnosed as having myeloproliferative syndrome (MPS) and had a trisomy 8 in all bone marrow cells. Five other MDS patients were used in a second screening to confirm differential expression of partial cDNA sequences. Two of them had RA (UPN 4 and 6), two RA with excess of blasts (RAEB, UPN 3 and 7), and one was unclassified (UPN 5). UPN 3, 5, and 6 were cytogenetically normal, whereas UPN 4 and 7 had deletions of the long arm of chromosome 5 in $90-100 \%$ of their bone marrow cells.

\section{Cell separation and RNA extraction}

Thirty milliters of EDTA blood was used to obtain PMNs by Ficoll-isopaque density gradient separation $\left(1.077 \mathrm{~g} / \mathrm{cm}^{2}\right)$. Total cellular RNA was isolated from these cells by the guanidium-thiocyanate-phenol-chloroform extraction procedure, as described previously [4]. In case RNA was isolated from fewer than $1 \times 10^{6}$ cells, $25 \mu \mathrm{g}$ glycogen was added as a carrier.
The chromosomal DNA was removed by incubation for $30 \mathrm{~min}$ at $37^{\circ} \mathrm{C}$ with 10 units of ribonuclease inhibitor (BRL, Gaitherburg, Md., USA), 10 units DNAse I (BRL) in $10 \mathrm{~m} M$ Tris$\mathrm{HCl} \mathrm{pH} 8.3,50 \mathrm{~m} M \mathrm{KCl}, 1.5 \mathrm{~m} M \mathrm{MgCl}_{2}$. After extraction with phenol/ $\mathrm{CHCl}_{3}(3: 1)$, the RNA in the aqueous layer was precipitated and dissolved in water.

\section{Differential display PCR}

cDNA subsets were prepared using three of the 12 possible anchored poly $(\mathrm{dT})_{12}$ primers $\left(\mathrm{T}_{12} \mathrm{AG}, \mathrm{T}_{12} \mathrm{CC}\right.$, and $\left.\mathrm{T}_{12} \mathrm{GC}\right)$. The reactions were performed as described by Liang and Pardee [10], with some minor modifications. For each cDNA synthesis, RNA isolated from $2 \times 10^{4}$ PMNs were reverse transcribed using 10 units of MMLV reverse transcriptase (BRL) for $60 \mathrm{~min}$ at $37^{\circ} \mathrm{C}$. The reaction mixture $(20 \mu \mathrm{l})$ further contained $1 \mu M$ of one of the anchored poly $(\mathrm{dT})_{12}$ primers, $20 \mu M$ dNTP, $50 \mathrm{~m} M$ Tris, $75 \mathrm{~m} M$ $\mathrm{KCl}, 3 \mathrm{mM} \mathrm{MgCl} 2$, and $10 \mathrm{~m} M$ DTT, $\mathrm{pH} 8.3$.

The PCR amplifications ( $20 \mu \mathrm{l}$ each) were performed on $2 \mu \mathrm{l}$ of heat-inactivated reverse transcriptase mixture, using combinations of an arbitrary 10-mer primer and the appropriate anchored poly (dT) $)_{12}$ primer $(1 \mu M$ each $)$, in the presence of $2 \mu M$ of each dNTP, $0.25 \mu M\left[\alpha^{-}{ }^{35} \mathrm{~S}\right] \mathrm{dATP}(1000 \mathrm{Ci} / \mathrm{mmol}$; Amersham, UK), $2 \mathrm{mM} \mathrm{MgCl} 2,50 \mathrm{mM} \mathrm{KCl}, 10 \mathrm{~m} M$ Tris- $\mathrm{HCl}$ (pH 8.4), 0.02\% BSA, and 1 unit of Taq polymerase (AmpliTaq; Cetus, Calif., USA). Following an initial denaturation at $95^{\circ} \mathrm{C}$ for $5 \mathrm{~min}$, the samples were amplified for 40 cycles of incubation at $95^{\circ} \mathrm{C}$ for $0.5 \mathrm{~min}$, $40^{\circ} \mathrm{C}$ for $2 \mathrm{~min}$, and $72^{\circ} \mathrm{C}$ for $1 \mathrm{~min}$. The reaction products were analyzed on $6 \%$ polyacrylamide (PAA) denaturing gels. The dried gels were exposed for at least $16 \mathrm{~h}$ to $\mathrm{X}$-ray films.

\section{Verification of differential expression of cDNA fragments}

First, the specificity was tested by repeating the DDPCR on newly prepared cDNA. The partial cDNAs that were still differentially expressed were excised from the dried gels, boiled in $100 \mu \mathrm{l}$ $\mathrm{H}_{2} \mathrm{O}$ for $10 \mathrm{~min}$, and re-amplified, using the conditions described above, but now in the presence of $2.5 \mathrm{~m} M$ dNTP and without $[\alpha-$ ${ }^{35}$ S]dATP. These PCR products were cloned into pCR1000 using the TA cloning system (Invitrogen, San Diego, Calif., USA). To check the size of the recombinant clones, the inserts were reamplified for ten cycles and analyzed on denaturing gels, which also contained the original display PCR samples from the patients and controls. Only the cloned fragments that co-migrated with the differentially expressed fragments were sequenced using a sequence kit (Boehringer Mannheim Biochemicals, Mannheim, Germany) according to descriptions provided by the supplier. Computer searches against the sequences in database GenBank (dated January 1,1996$)$ were performed to check for homology. Based on the sequence, nested 20-mer primers were synthesized that allowed verification of the differential expression by PCR amplification using the specific primer sets. For this purpose, cDNA was prepared by reverse transcription with poly $(\mathrm{dT})_{18}$ from $2 \mu \mathrm{g}$ RNA of the same patient, the patients of the second screening panel, and control samples.

The chromosomal localization of individual partial cDNAs was determined by PCR analysis of the human-rodent somatic cell hybrid mapping panel 2 (NIGMS), using the amplicon-specific primers. This panel consists of DNA isolates from 24 hybrids, each retaining a single intact human chromosome.

\section{Results}

Validation of the DDPCR method using small cell numbers

The differential display technique was originally described to detect differently expressed genes from cell 
lines [10]. We have used purified PMNs to compare expression patterns between MDS patients and healthy controls. Due to the limited number of patient cells available, the procedure had to be scaled down with respect to cell numbers and amount of RNA that could be analyzed. RNA was isolated from $1 \times 10^{2}$ to $1 \times 10^{6}$ cells in the presence of $25 \mu \mathrm{g}$ tRNA or $25 \mu \mathrm{g}$ glycogen. Reproducible patterns on denaturing PAA gels were obtained when at least $2 \times 10^{4}$ cells were used. This is one order of magnitude lower than originally described, assuming that $0.02 \mu \mathrm{g}$ RNA can be isolated from these cells. When lower cell numbers were used, patterns of lower complexity were obtained in each PCR reaction. Also at the level of PCR amplification, a tenfold dilution of the cDNA samples was used effectively without loss of information. Equally good results were obtained with both types of carriers. For further experiments glycogen was used because of the potential interference of tRNA in the amplification step.

Initial screening of MDS samples using differential display PCR

Using the adaptations described above, total RNA samples from PMNs of three healthy individuals and of the two MDS patients were analyzed. To date, 20 combinations of arbitrary 10 -mer and anchored poly $(\mathrm{dT})_{12}$ primers have been tested. Each primer combination revealed 50-100 different PCR fragments on denaturing PAA gels. The expression profiles for the individual PCR reactions were largely identical between the patient and control samples. Only minor differences were observed (Fig. 1). Five partial cDNA sequences were identified which were either absent or present in both MDS patients as compared with the healthy controls (see, for example, Fig. 2a), while three other fragments were differentially expressed in individual patients (Fig. 2b).

It should be noted that the fragments were often present as doublets in the denaturing PAA gels. Sequence analysis of both fragments and gel migration analysis of the cloned fragments (see below) revealed that this phenomenon is due to small differences in the electrophoretic mobility of the two complementary DNA strands.

Analysis of the authenticity of aberrantly expressed fragments

Because of the limited amount of RNA available, we were not able to perform Northern analysis to unambiguously show aberrant expression of a given fragment. Several PCR-based control experiments were therefore included to check the authenticity of differential expression. The specificity was first verified by reanalyzing the same primer combinations on newly prepared
Subset 1

$\begin{array}{llllll}1 & 2 & 3 & 4 & 5\end{array}$

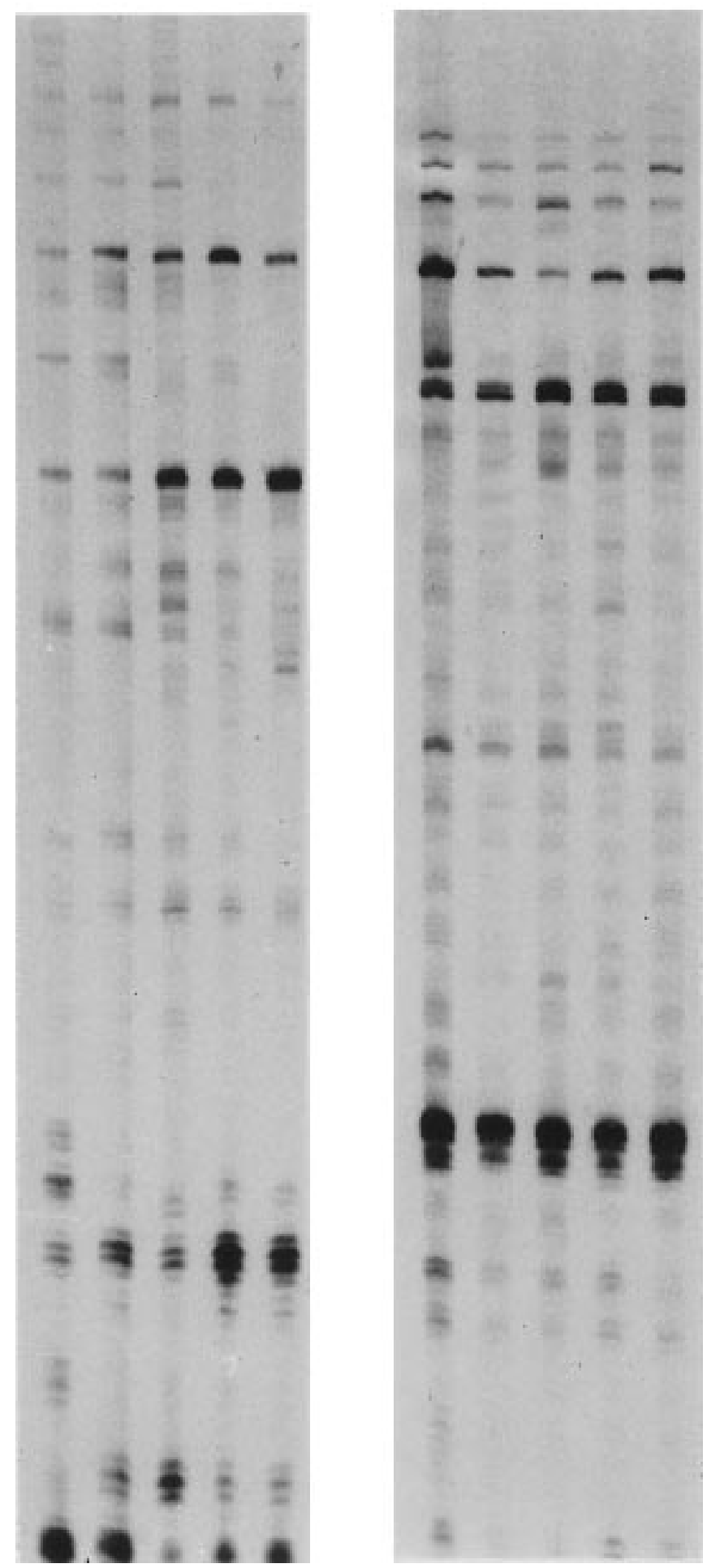

Fig. 1 Display of partial cDNAs on 6\% PAA denaturing gels for two primer combinations (subsets 1 and 2). Lanes 1-3, PMNs of healthy controls; lanes 4, 5, PMNs from MDS patients 
Fig. 2a, b Examples of differentially expressed cDNA fragments. a DC5 (arrow) is present in both patients, UPN 1 $(P 1)$ and UPN $2(P 2)$, but absent in the healthy controls (C1 and C2). b DC3 (arrows) is absent in UPN 1, but present in UPN 2 and the healthy controls


cDNAs. Two fragments (DC5, $467 \mathrm{bp}$, and DC7, $393 \mathrm{bp}$ ) were reproducibly aberrantly expressed in both MDS patients, whereas one cDNA fragment (DC3, $215 \mathrm{bp}$ ) was reproducibly absent in the patient with a $5 q$ deletion (UPN 1). For further characterization, the individual bands were recovered from the dried sequencing gels, reamplified using the corresponding primer sets, and cloned into the pCR1000 plasmid vector. To eliminate false-positive clones, they were first checked by fractionation on denaturing PAA gels alongside the original DDPCR products. For example, in case of DC3, only three of six clones tested were of the correct length. Clones containing the DC5, DC7, and DC3 PCR products were subsequently sequenced (e.g., Fig. 3). Genbank searches revealed that DC5 was identical to an anonymous fragment isolated from a muscle cDNA library. For the other two fragments no similarities were found; therefore, they could represent uncharacterized genes. All fragments were flanked by the arbitrary 10 -mer primers, rather than by a 10 -mer and an anchored poly $(\mathrm{dT})_{12}$ primer. This is possibly due to the ratio between the arbitrary primer and anchored poly $(\mathrm{dT})_{12}$ used, which was fivefold higher, as described previously [10].
For the three remaining fragments, amplicon-specific primer sets (20-mers) were synthesized (e.g., Fig. 3, underlined). These nested primers were used to ascertain the differential expression by amplification of poly $(\mathrm{dT})_{18} \mathrm{cDNA}$ from the same patient, two control samples, and the five MDS patients of the secondary panel. Only in the case of DC3 it was possible to confirm differential expression in this way. The amplicon was present in PMNs from normal controls, and decreased in intensity or barely detectable in those from five of the

TTAGATGTGA GTTAATTGCT GACCATGTGG ATTTAGAATC TGTTTTAAAC TGGGTGCTAT TTAGAAGACA GTGGGTCTTC ATTGGTTGCT GGTTTTAGTA TCTAACGAAA CCAGTTTTAG TTTTTAGACC AGTATAGCTA TCTTGAGAAA TTAACTTTCG

\section{GAGTCCTATG TCGGATACCT GGGGC $\underline{\underline{T G G G C ~ T C A T G}}$}

Fig. 3 Partial nucleotide sequence of the DC 3 cDNA clone. The arbitrary 10-mer primer used is underlined twice and the amplicon-specific 20-mer primers are underlined once 


\section{- C1 C2 P3 P1 P4 P5 P6 P7}

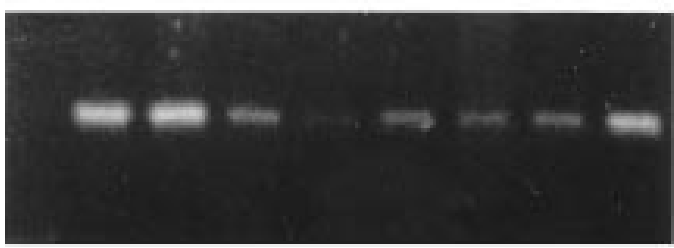

DC3

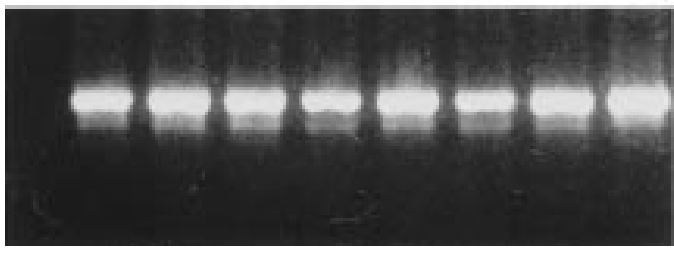

HPRT

Fig. 4 Nested PCR with amplicon-specific primers to confirm the differential expression of DC3 in PMNs of two healthy controls $(C 1, C 2)$, UPN $1(P 1)$, and the five other MDS patients (P3-P7)

six MDS patients after 35 rounds of amplification (Fig. 4).

The chromosomal localization of DC3 was determined by PCR of DNA isolates from 24 hybrid cell lines of a mapping panel with the specific 20-mers. This revealed that DC3 localizes to chromosome 3 (data not shown).

\section{Discussion}

Only a handful of genes involved in the pathogenesis of MDS have been identified. In the majority of cases, these genes were implicated in structural chromosomal aberrations such as translocations and inversions and were identified following labor-intensive molecular genetic approaches. For the numerical abnormalities observed in MDS these procedures are not applicable.

Recently, the DDPCR technique has been developed, allowing direct comparison of expression patterns between tumor and normal cells. This procedure has proven useful for the isolation of differentially expressed genes in several cell lines derived from tumors $[14,15]$. We have tested whether the method could also be applied to purified primary cells from MDS patients. This meant that the procedure had to be scaled down with respect to the cell numbers required. Furthermore, several controls (preferably PCR based) were included to verify aberrant expression of the partial cDNAs detected during the primary screens. For example, RTPCR with fragment-specific nested primers was performed, rather than Northern hybridization. Another important issue was the elimination of false-positive fragments following cloning of differentially expressed fragments through fractionation on denaturing PAA gels alongside the original DDPCR sample. An alternative procedure has been described by Callard et al. [3], who reduced the number of contaminating sequences by hybridization of the purified PCR products to dotblots containing the cloned fragments.

We have compared the expression patterns of purified PMNs from two MDS patients with those of healthy individuals. The expression fingerprints obtained with individual primer combinations were almost identical for the different samples. So far, 20 primer combinations have been tested in this pilot study. If we assume that a given cell type expresses 15000 different mRNAs on average, and one PCR amplification generates 50100 cDNA fragments, about $1 / 10$ mRNAs expressed in PMNs have been screened. Of them, three were consistently aberrantly expressed, either in both patients (DC5 and DC7) or in an individual patient (DC3).

The decreased expression of DC3 in the patient with the $5 q$ deletion (UPN 1) was confirmed by PCR with specific primers on poly(dT) cDNA. Also in the secondary panel of MDS patients the expression level was decreased or barely detectable in most of the samples, indicating that this gene could be disregulated in MDS. Altered RNA expression levels in a subset of MDS patients have been observed for a variety of genes, such as multidrug resistance P-glycoprotein [16], c-mpl [19], c-fms, c-kit, and flt3 [2]. We can assume that the majority of them represent a common consequence of dysregulated growth and are not directly involved in leukemogenesis. DC3 maps to chromosome 3, as was shown using the human-rodent hybrid mapping panel. This localization could be of interest, since several known MDS-related genes, such as Evi-1, EAP, and MDS-1, are known to be clustered here [12, 13].

In conclusion, differential display PCR has proven to be an elegant procedure that allows simultaneous assessment of multiple primary patient samples to identify aberrantly expressed cDNA sequences. With respect to DC3, isolation of the full-length cDNA could provide insight into its potential role in the pathogenesis of MDS.

\section{References}

1. Bennett JM, Catovsky D, Daniel MT, Flandrin G, Galton DAG, Gralnick HR, Sultan C, the FAB Co-operative group (1982) Proposals for the classification of the myelodysplastic syndromes. Br J Haematol 51:189-199

2. Birg F, Rosnet O, Carbuccia N, Birnbaum D (1994) The expression of fms, kit and flt 3 in hematopoietic malignancies. Leuk Lymphoma 13:223-337

3. Callard D, Lescure B, Mazzolini L (1994) A method for the elimination of false positives generated by the mRNA differential display technique. BioTechniques 16:1096-1103

4. Falkenburg JHF, Harrington MA, De Paus RA, Walsh WK, Daub R, Landegent JE, Broxmeyer HE (1991) Differential transcription and post-transcriptional regulation of gene expression of the colony-stimulating factors by interleukin-1 and fetal bovine serum in murine fibroblasts. Blood 78:658665

5. Gerritsen WR, Donohue J, Bauman J, Jhanwar SC, Kernan NA, Castro-Malaspina H, O'Reilly RJ, Bourhis J-H (1992) Clonal analysis of myelodysplastic syndrome: monosomy 7 is expressed in the myeloid lineage, but not in the lymphoid lineage as detected by fluorescent in situ hybridization. Blood $80: 217-224$ 
6. Kibbelaar RE, van Kamp H, Dreef EJ, Groot-Swings G, Kluin-Nelemans JC, Beverstock GC, Fibbe WE, Kluin PhM (1992) Combined immunophenotyping and DNA in situ hybridization to study lineage involvement in patients with myelodysplastic syndromes. Blood 79:1823-1828

7. Kroef MJPL, Fibbe WE, Mout R, Jansen RPM, Haak HL, Wessels JW, Van Kamp H, Willemze R, Landegent JE (1993) Myeloid but not lymphoid cells carry the $5 \mathrm{q}$ deletion: polymerase chain reaction analysis of loss of heterozygosity using mini-repeat sequences on highly purified cell fractions. Blood $81: 1849-1854$

8. Le Beau MM, Espinosa III R, Neuman WL, Stock W, Toulston D, Larson RA, Keinanen M, Westbrook CA (1993) Cytogenetic and molecular delineation of the smallest commonly deleted region of chromosome 5 in malignant myeloid diseases. Proc Natl Acad Sci USA 90:5484-5488

9. Lee SW, Tomasetto C, Sager R (1991) Positive selection of candidate tumor-suppressor genes by subtractive hybridization. Proc Natl Acad Sci USA 88:2825-2829

10. Liang P, Pardee AB (1992) Differential display of eukaryotic messenger RNA by means of the polymerase chain reaction. Science 257:967-971

11. Mufti GJ (1992) Chromosomal deletions in the myelodysplastic syndrome. Leuk Res 16:35-41

12. Nucifora G, Begy CR, Kobayshi H, Roulston D, Claxton D, Pederson-Bjergaard J, Parganas E, Ihle JN, Rowley JD (1994) Consistent intergenic splicing and production of multiple transcripts between AML1 at 21q22 and unrelated genes at $3 \mathrm{q} 26$ in $(3 ; 21)(\mathrm{q} 26 ; \mathrm{q} 22)$ translocations. Proc Natl Acad Sci USA 91:4004-4008

13. Russell M, List A, Greenbert P, Woodward S, Glinsmann B, Parganas E, Ihle J, Taetle R (1994) Expression of EVI1 in myelodysplastic syndromes and other hematologic malignancies without 3q36 translocations. Blood 84:1243-1248
14. Sager R, Anisowicz A, Neveu M, Liang P, Sotiropoulou G (1993) Identification by differential display of alpha 6 integrin as a candidate tumor suppressor gene. FASEB J 7:964-970

15. Shinoura N, Shamraj OI, Hugenholz H, Guang Zhu J, McBlack P, Warnick R, Tow JJ, Wani MA, Menon AG (1995) Identification and partial sequence of a cDNA that is differentially expressed in human brain tumors. Cancer Lett $89: 215-221$

16. Sonneveld P, van Dongen JJM, Hagemeijer A, van Lom K, Nooter K, Schoester M, Adriaansen HJ, Tsuruo T, de Leeuw K (1993) High expression of the multidrug resistance P-glycoprotein in high risk myelodysplasia is associated with immature phenotype. Leukemia 7:963-969

17. Tefferi A, Thibodeau SN, Solberg LA (1990) Clonal studies in myelodysplastic syndrome using X-linked restriction fragment length polymorphisms. Blood 75:1770-1773

18. van Kamp J, Fibbe WE, Jansen RPM, van der Keur M, de Graaff E, Willemze R, Landegent JE (1992) Clonal involvement of granulocytes and monocytes, but not of T and B lymphocytes and natural killer cells in patients with myelodysplasia: Analysis by X-linked restriction fragment polymorphisms and polymerase chain reaction of the phosphoglycerate kinase gene. Blood 80:1774-1778

19. Vigon I, Dreyfus F, Melle J, Viguie F, Ribrag V, Cocault L, Souyri M Gisselbrecht S (1993) Expression of the c-mpl proto-oncogene in human hematologic malignancies. Blood $82: 877-883$

20. Zou Z, Anisowicz A, Hendrix MJC, Thor A, Neveu M, Sheng S, Rafidi K, Seftor E, Sager R (1994) Maspin, a serpin with tumor-suppressing activity in human mammary epithelial cells. Science 263:526-529 